\title{
The Ginzburg regime and its effects on topological defect formation
}

\author{
Luís M. A. Bettencourt ${ }^{1}$, Nuno D. Antunes ${ }^{2}$, and W.H. Zurek ${ }^{1}$ \\ ${ }^{1}$ T-6, Theoretical Division, Los Alamos National Laboratory, Los Alamos NM 87545, USA \\ ${ }^{2}$ Blackett Laboratory, Imperial College, London SW7 2BZ, United Kingdom.
}

(October 27, 2018)

\begin{abstract}
The Ginzburg temperature has historically been proposed as the energy scale of formation of topological defects at a second order symmetry breaking phase transition. More recently alternative proposals which compute the time of formation of defects from the critical dynamics of the system [1], have been gaining both theoretical and experimental support. We investigate, using a canonical model for string formation, how these two pictures compare. In particular we show that prolonged exposure of a critical field configuration to the Ginzburg regime results in no substantial suppression of the final density of defects formed. These results dismiss the recently proposed role of the Ginzburg regime in explaining the absence of topological defects in ${ }^{4} \mathrm{He}$ pressure quench experiments.
\end{abstract}

PACS Numbers : 05.70.Fh, 11.27.+d, 98.80.Cq

LAUR-99-6369

\section{INTRODUCTION}

The Ginzburg temperature $T_{G}$ [1] was thought historically to be the determining energy scale at which topological defects are formed in the aftermath of a second order symmetry breaking phase transition 12 - 4 . More recently theoretical and experimental progress has pointed in the direction that it is the critical dynamics of the second order transition, induced by a change in some external parameter such as temperature or pressure, that determines the numbers (and configuration) of topological defects emerging below the critical point 5 .

Nevertheless the role of large thermal fluctuations within the Ginzburg regime in defect formation mechanisms remains relatively poorly understood. In particular it is not clear how a density of defects created, presumably by the critical dynamics of the system, could evade alteration when exposed extensively to thermal fluctuations in the Ginzburg regime.

This issue has been rekindled recently due to the possibility of new experimental tests [6 9] and, in particular, by the negative results of a pressure quench experiment in ${ }^{4} \mathrm{He}$ [9], a system in which (because of strong interactions) the Ginzburg regime is particularly extensive. This experiment has improved on an apparatus used earlier by McClintock et al. [] to implement a superfluid transition in ${ }^{4} \mathrm{He}$ through a sudden pressure quench. The results show no evidence for the formation of topological defects at the anticipated levels, contrary to expectations based both on the old experiment [8], the theory $]^{\text {and }}$ and ${ }^{3} \mathrm{He}$ data 6.7].

\footnotetext{
*Although a factor $f \gtrsim 10$ in the formula for the string density $n \sim 1 /(f \hat{\xi})^{2}$ could explain the new results and seems consistent with recent numerical studies 10$]$.
}

The discrepancy with the earlier ${ }^{4} \mathrm{He}$ quench data is now seen as the evidence of mechanical stirring in the first version of the experiment. Nevertheless to address this discrepancy with ${ }^{3} \mathrm{He}$ it was suggested [11] that because the Ginzburg regime in ${ }^{4} \mathrm{He}$ extends over a broad range of temperatures around the $\lambda$-line, large scale fluctuations may be able to unwind and alter the configuration of the order parameter (in contrast to ${ }^{3} \mathrm{He}$ ) while the quench proceeds. The Ginzburg temperature is defined, somewhat qualitatively, through the loss of ability of the order parameter to hop, through thermal activation, over the potential barrier between broken symmetry minima. Thus one might worry with Karra and Rivers 11] that when the defect densities are eventually measured, at a much later time, little or no string would have survived unwinding through thermal activation.

In in this paper we study in detail the role of the Ginzburg regime in vortex string formation. In section II we discuss our model and its properties. We show in particular that it transcends the more usual time dependent Ginzburg-Landau (TDGL) dynamics in generality and reduces to it in particular cases. In section III we describe the traditional arguments for the existence of a well defined Ginzburg temperature and critically analyze their underlying assumptions in the light of known results on the thermodynamics of vortex strings. We also establish a quantitative definition of the Ginzburg temperature and discuss its uncertainties. In section [IV] we investigate the role of the Ginzburg regime in the dynam$i c s$ of defect formation. This is achieved by exposing field configurations created at criticality to intermediate temperatures within the Ginzburg regime and analyze the effect upon the final density of defects measured at late times. We also study the memory of the order parameter when subjected to reheating. This constitutes a direct test on the theory of defect formation as a consequence of the critical dynamics of the theory. Finally we draw 
our conclusions and discuss in the light of our results the possible relevance of the Ginzburg regime in explaining recent experimental results in ${ }^{4} \mathrm{He}$ pressure quench experiments.

\section{LANGEVIN AND FOKKER-PLANCK FIELD DYNAMICS}

As a working model we consider a $U(1)$ symmetric $\lambda \phi^{4}$ global field theory in 3 spatial dimensions $(3 \mathrm{D})$, in the canonical ensemble, i.e. in contact with a heat bath at a given temperature $T$. This model has the advantage of having been extensively studied in thermal equilibrium 13, 14, 12, 15 and moreover of describing the thermodynamics of ${ }^{4} \mathrm{He}$ at criticality 16] by permitting the computation of relevant critical exponents with extreme accuracy.

As such it supplies us with a controlled realistic environment in which the role of thermal fluctuations within the Ginzburg regime in changing the density of topological defects can be studied. The equations of motion for the fields are

$$
\begin{aligned}
& {\left[\partial_{t}^{2}+\eta \partial_{t}-\nabla^{2}-m^{2}\right] \phi_{i}(x)} \\
& \quad+\lambda\left(\sum_{j=1}^{2} \phi_{j}^{2}(x)-1\right) \phi_{i}(x)=\xi_{i}(x, t), \\
& \left\langle\xi_{i}(x, t)\right\rangle=0, \\
& \left\langle\xi_{i}(x, t) \xi_{j}\left(x^{\prime}, t^{\prime}\right)\right\rangle=\Omega \delta\left(x-x^{\prime}\right) \delta\left(t-t^{\prime}\right) \delta_{i j} .
\end{aligned}
$$

where $i, j \in\{1,2\}$ and the heat bath fields $\xi_{i}(x, t)$ obey the fluctuation dissipation relation in equilibrium. Thus, for long times, the system equilibrates to its canonical distribution at temperature $T$. This can be shown most conveniently by writing the Fokker-Planck equation corresponding to the Langevin dynamics of Eq. (11) [17],

$$
\partial_{t} P_{F P}[\pi, \phi, t]=-\mathcal{H}_{\mathrm{FP}} P_{F P}[\pi, \phi, t] .
$$

where

$$
\begin{aligned}
\mathcal{H}_{\mathrm{FP}}=-\frac{\Omega}{2} \frac{\delta^{2}}{\delta \pi_{i}^{2}} & +\pi_{i} \frac{\delta}{\delta \phi_{i}} \\
& -\frac{\delta}{\delta \pi_{i}}\left(\eta \pi_{i}-\nabla^{2} \phi_{i}+\frac{\delta V(\phi)}{\delta \phi_{i}}\right),
\end{aligned}
$$

where sum over $i \in\{1,2\}$ is implied here and below. If, as in most applications, the potential $V(\phi)$ is explicitly time independent we can invoke a separation ansatz for $P_{F P}$ such that

$$
P_{F P}[\pi, \phi, t]=\mathcal{P}[\pi, \phi] T(t)
$$

Thus we can regard Eq. (2) as a functional Schrödinger equation, in imaginary time. Then we can write the time independent and dependent equations

$$
\mathcal{H}_{\mathrm{FP}} \mathcal{P}_{n}=E_{n} \mathcal{P}_{n}, \quad \partial_{t} T(t)=-E_{n} T(t)
$$

The functional dependence on the fields is now limited to the static probability eigenfunctionals $\mathcal{P}_{n}$. The time evolution of the Fokker-Planck distribution is completely characterized by the spectrum of eigenvalues of $\mathcal{H}_{\mathrm{FP}}, E_{n}$.

Formally, we can then project the evolution of $P_{F P}$ in terms of its eigenvalues $E_{n}$ and eigenfunctionals $\mathcal{P}_{n}$ as:

$$
P_{F P}[\pi, \phi, t]=\sum_{n=0}^{\infty} C_{n} \mathcal{P}_{n}[\pi, \phi] e^{-E_{n} t} .
$$

where the $C_{i}$ 's are the projections of the initial time $P_{F P}$ onto the basis of eigenfunctionals $\mathcal{P}_{n}$.

The equilibrium solution must be static. It corresponds to a zero eigenvalue in Eq. (5), which implies the solution

$P_{\text {eq }}[\pi, \phi]=N \exp \left[-\beta \int d^{D} x \frac{\pi_{i}^{2}}{2}+\frac{\left(\nabla \phi_{i}\right)^{2}}{2}+V[\phi]\right]$,

where we took $\Omega=2 \eta / \beta$, which is the Einstein relation enforcing equilibrium between fluctuation and dissipation at long times. Summation over $i$ is implied. On general grounds we expect the canonical equilibrium distribution to be approached at long times, i.e. we expect the excited time-dependent states $P_{n}, n \neq 0$ to decay with time.

The full spectrum of excited states and their corresponding eigenvalues can only be found for specific forms of the field potential $V(\phi)$. In particular this is possible in closed analytic form for harmonic potentials $V=\frac{1}{2} m^{2} \phi^{2}$. For each mode in k-space the excited states are given in terms of Hermite polynomials of functions of the field modes and those of their conjugate momenta. More importantly the corresponding eigenvalues are given by 17

$$
E_{n}=n \frac{\eta}{2}\left[1 \pm \sqrt{1-4\left(k^{2}+m^{2}\right) / \eta^{2}}\right]
$$

Close to the phase transition the leading effect of the $\frac{\lambda}{4} \phi^{4}$ interactions is to make the effective mass temperature dependent as

$$
m^{2}(T)=m_{0}^{2}\left|\frac{T-T_{c}}{T_{c}}\right|^{2 \nu}
$$

where $T_{c}$ is the critical temperature and $\nu$ a universal critical exponent, which depends only on the dimensionality of space and the internal symmetries of the theory. To 1-loop in perturbation theory we have

$$
\begin{array}{r}
m^{2}(T)=-m^{2}+\Delta m^{2}(T), \\
\Delta m^{2}(T)=\lambda \int \frac{d^{D} k}{(2 \pi)^{D}}\left\langle\phi_{k} \phi_{-k}\right\rangle .
\end{array}
$$

i.e. the temperature correction to $m^{2}$ is given by the (classical) tadpole diagram. The values of the $O(2)$ symmetric thermal average $\left\langle\phi_{k} \phi_{-k}\right\rangle$ depends on the specific 
form of the thermal distribution, classical or quantum. Under these approximations one obtains the mean-field value of $\nu=1 / 2$.

In the critical domain we can thus obtain an approximate analytical description of the non-linear field dynamics by taking the mass in the harmonic potential to be of the form (8). Although only approximately true we will show below that this assumption leads to a good qualitative understanding of the full non-linear field dynamics in the critical domain and the effects of the Ginzburg regime.

The present scheme, Eqs. (1), is therefore convenient both as a thermalization algorithm, if the system is started at any given configuration and run for long times, or as a means of performing effective non-equilibrium dynamics. The equilibration time itself $t_{e q} \simeq 1 / E_{1}$, is dependent on spatial scale (or wave length) and on temperature. Qualitatively large spatial scales equilibrate more slowly and in particular, in the critical domain, exhibit critical slowing down. This can be seen explicitly by considering long wave-length modes $\left(k^{2} \simeq 0\right)$ in the vicinity of $T_{c}$. Then, for half of the eigenvalues the termalization time is inversely proportional to $n$ times $t_{e q}$ with

$$
\begin{aligned}
t_{e q}= & {\left[\frac{\eta}{2}\left(1-\sqrt{1-4\left(k^{2}+m^{2}\right) / \eta^{2}}\right)\right]^{-1} } \\
& \simeq \frac{\eta}{m^{2}(T)} \rightarrow \infty,
\end{aligned}
$$

as $T \rightarrow T_{c}$. This is the result for overdamped dynamics $\eta>>m(T)$, and could have been obtained by a Langevin equation with a single (dissipative) time derivative. In this sense the evolution of the long-wave length modes in the vicinity of $T_{c}$ is always overdamped, which is the essence of the perhaps more familiar TDGL evolution, to which Eqs. (1) reduce to in the appropriate regime. Note that the TDGL dynamics is an effective equation for long-wave length field modes in the critical domain while our model holds more generally.

In the converse limit the decay of short wave-length modes is dictated by $\eta$ and is thus scale invariant in this approximation. The appropriate physical value of $\eta$ can be computed in perturbation theory given a microscopic model. Particularly interesting are situations for which $m(T)<\eta$ as happens in the critical domain. Then there is true time-scale separation in the sense that short wavelength modes thermalize much faster than long-wave length modes.

This kind of considerations will help us understand the behavior of the fully non-linear dynamics in the Ginzburg regime. Before we do this we need to develop a clear picture of equilibrium to which we now turn.

\section{EQUILIBRIUM RESULTS AND THE DEFINITION THE GINZBURG TEMPERATURE}

The rationale behind the original proposal according to which the Ginzburg temperature $T_{G}$ is the energy scale for the formation of topological defects [2, 3], was that, at lower temperatures, thermal fluctuations would be unable to overcome the potential energy barrier associated with the defect's topological stability. Thus, field configurations with non-trivial topology, below this temperature would necessarily acquire stability on the average.

It is clear that such an appealingly simple physical picture assumes implicitly a separation of physical scales and associated time evolution or equivalently, as we discuss below, that at least part of the system is out of thermal equilibrium. Indeed one must assume that field configurations can be separated in two populations - one of topological defects and another of thermal fluctuations. The former, at least in the sense of the definition of the Ginzburg regime (see below) live on a zero temperature background. This situation is at best an idealization.

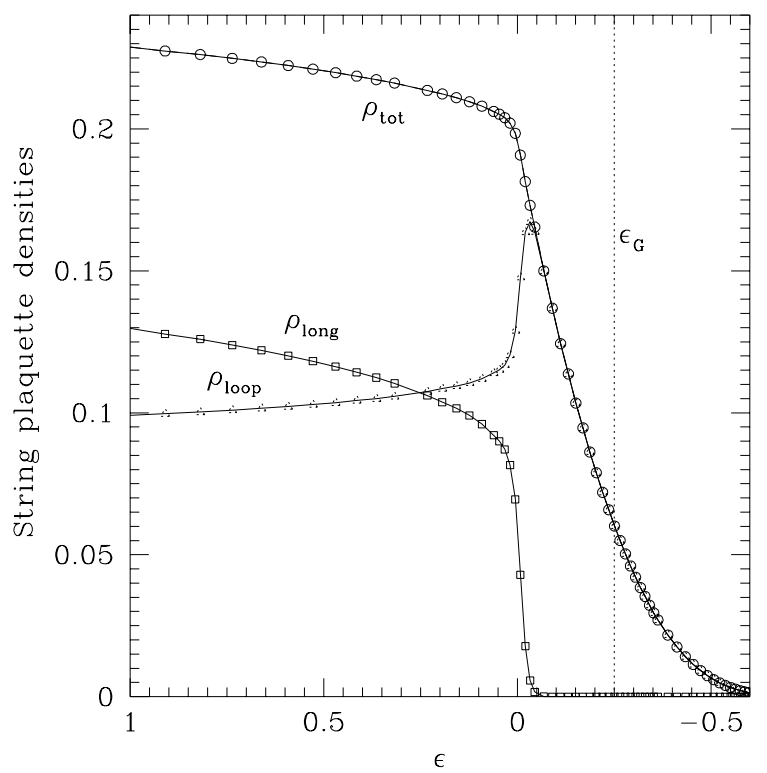

FIG. 1. The dependence of the thermal distribution of vortex string field fluctuations on temperature $\left(\epsilon=\frac{T-T_{c}}{T_{c}}\right)$, around the critical point $\epsilon=0$. The critical point is marked by the sudden appearance of long strings. The observed fast transient should become a discontinuous jump in the infinite volume limit [16].

Strictly in thermal equilibrium at temperatures not too low, field thermal fluctuations with non-trivial topology will always exist. The density of vortex string thermal fluctuations in our model is shown in Fig. 1. It is, however, remarkable that long strings can only exist in equilibrium strictly above $T_{c}$ 13,14. This phenomenon is the analog of vortex pair unbinding in the well known Kosterlitz-Thouless transition in this very same model in 2D 18,19]. In 3D, however, long strings appear abruptly as we are dealing with a true critical phenomenon instead of a crossover.

The appearance of long strings exactly at $T_{c}$ can be understood, in turn, in terms of their thermal statisti- 
cal properties namely their tension $\sigma_{\text {eff }}(T)$ (free energy per unit length) and other statistical properties like their fractal or Hausdorf dimension [14]. The dependence of the string tension on temperature is shown in Fig. 2. As seen the string tension diminishes continuously with increasing temperature until the critical point where it vanishes. This permits the creation of strings of all lengths above $T_{c}$. Below $T_{c}$, on the other hand strings are exponentially suppressed and only those smaller than the temperature dependent length $l \simeq T / \sigma_{\text {eff }}(T)$ are likely as thermal fluctuations. It is the existence of long strings as thermal fluctuations that will lead to defect formation if the system is suddenly cooled [10].

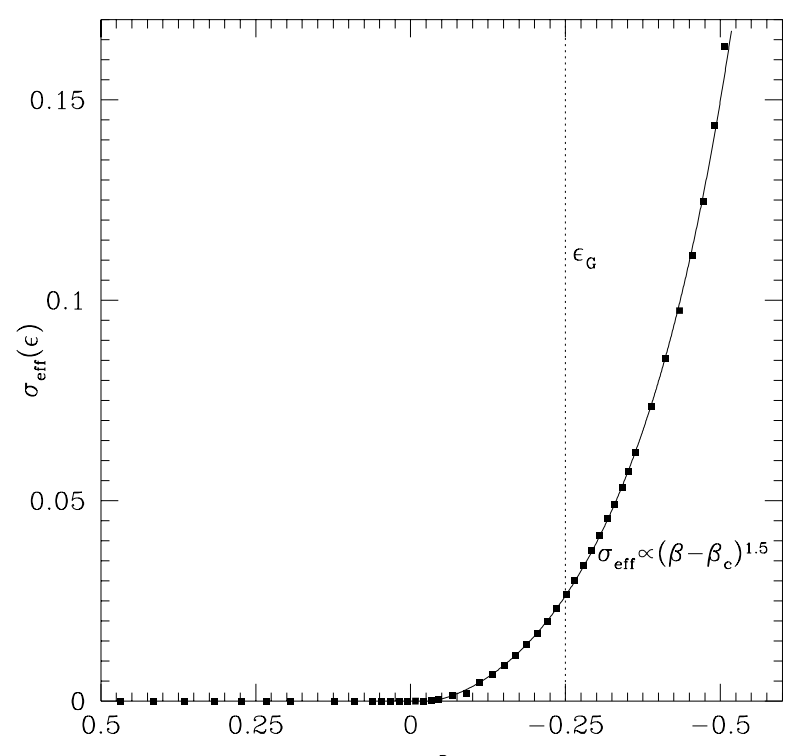

FIG. 2. The dependence of the string tension $\sigma$ (free energy per unit length) on temperature in the critical domain. The critical point is marked by the vanishing of $\sigma$, which in turn allows for the creation of arbitrarily long strings as thermal fluctuations, see Fig. 1.

Although some of the above comments may seem somewhat marginal they establish that the thermodynamics of the $U(1)$ theory under consideration is much richer than the assumptions on which the traditional role of the Ginzburg regime is based. They show in particular that the vortex strings themselves as a subset of the theory's thermal fluctuations have a very non-trivial thermodynamics and cannot be taken as their cold classical solutions over a nontrivial thermal background.

The thermodynamics of vortex strings in more complex field theories, with gauge fields and larger symmetry groups, remains to date largely unstudied, although some work has been done in the Abelian case [20]. We expect nevertheless that most of the features of our $U(1)$ global theory may persist albeit characterized by different critical exponents. This statement is supported by analytic studies of the statistics of free strings [21,22], which, thanks to their large configurational entropy, exhibit a similar (Hagedorn) transition but display eg. a different dependence of the string tension on temperature. The interactions thus change particular temperature dependences of certain quantities but not their qualitative behavior. In theories where defects are not topologically stable however (as in the case of embedded or semilocal defects) the role of these configurations may be potentially different and possibly marginal.

The cumulative results from the equilibrium study of the thermodynamics of vortex strings in our model cast considerable doubt upon the assumptions underlying the traditional role of the Ginzburg temperature in defect formation. It remains unclear however what the role may be of large thermal fluctuations in the critical domain (above $T_{G}$ ) in changing defect densities produced by the critical dynamics of the fields.

In order to investigate this issue we need a quantitative definition of $T_{G}$. In tune with the arguments given above consider a volume of characteristic size $\xi(T)$, the correlation length, and a theory with two energetically degenerate minima of an effective potential $V(\phi)$, separated by a potential barrier $\Delta V$. This applies also for theories with a general $O(N)$ symmetry, since we will be interested in the 'radial' direction only. The effective potential is obtained by singling out an arbitrary direction in field space [23], which we denote by $\varphi$. The rate for the field to change coherently from one minimum to the other per unit volume due to thermal activation is

$$
T^{4} \exp \left(-\Delta V / k_{B} T\right)
$$

For an effective potential of the form (obtained, eg. perturbatively at 1-loop)

$$
V(\phi)=-\frac{1}{2} m^{2}(T) \varphi^{2}+\frac{\lambda}{4} \varphi^{4}
$$

$\Delta V=\frac{m(T)^{4}}{4 \lambda}$. For a volume $\xi^{3}$, we define $T_{G}$ such that the probability of overcoming the potential barrier is of order unity:

$$
T_{G}: \frac{\Delta V\left(T_{G}\right)}{T_{G}} \cdot \xi^{3}\left(T_{G}\right)=1 \Leftrightarrow \lambda T_{G} / m\left(T_{G}\right)=\frac{1}{4} .
$$

This definition however has some caveats, for instance, an effective potential of the form Eq. (13) is only valid for the mean field and not on smaller scales. A more careful accounting of scales leads to different results [24, which show an enhancement of the hoping probability. Thus, the factor of $1 / 4$ in Eq. (14) should not be taken at face value.

A perhaps more rigorous definition arises from the range of temperatures below $T_{c}$ for which fluctuations are large and consequently where perturbative finite temperature field theory fails to be useful. In order to set up a perturbative scheme at finite temperature from an initial $3+1$ dimensional quantum field theory one implements 
dimensional reduction which is valid provided the temperature is high compared to all mass scales. As a consequence the coupling of the dimensionally reduced 3D field theory becomes dimensionful, i.e. $\lambda \rightarrow \lambda T=\lambda_{3}$. In order to proceed one has to identify an appropriate dimensionless coupling. This is done by taking $\lambda T / m(T)$. The Ginzburg regime is entered when this $3 \mathrm{D}$ effective coupling becomes strong, in the vicinity of the critical point, namely

$$
T_{G}: \lambda T_{G} / m\left(T_{G}\right)=1
$$

To compute $T_{G}$ one needs the scaling of $m(T)$ in the critical domain. We write $m^{2}(T)=m_{0}^{2}|\epsilon|^{\nu}$, with $\epsilon$ being the reduced temperature $\epsilon=\frac{T-T_{c}}{T c}$.

Thus $\epsilon_{G}=-0.18$ for $\nu=0.5$. This mean-field estimate produces an upper bound in $T$ for $T_{G}$ (and lower bound for $\beta=1 / T)$. For realistic 3D exponents, $\nu=0.67$, we obtain $\epsilon_{G}=-0.25$. The first criterion, based on the hopping of a correlation sized volume, results in higher values of $T_{G}$. This brings about a relatively large uncertainty in the value of $T_{G}$, which is $18-25 \%$ below $T_{c}$.

\section{THE ROLE OF THE GINZBURG REGIME IN THE DYNAMICS OF DEFECT FORMATION}

In order to investigate the role of the Ginzburg temperature in the dynamics of defect formation we perform a series of tests both directly over the evolution of string densities and the evolution of the order parameter, when exposed to thermal fluctuations in the Ginzburg regime.

\section{A. Strings Survive the Ginzburg Regime}

To investigate the effects of thermal fluctuations directly upon strings we deliberately expose the system to a heat bath at temperature $\epsilon_{i}$, within the Ginzburg regime and below.

We are attempting to emulate the worst case scenario of an experimental quench where the temperature or pressure are dropped monotonically but where the system makes a long stopover within the Ginzburg regime. We repeat this procedure for a range of time intervals $\Delta t$, after which the bath temperature is taken to zero. This set of temperature trajectories is shown in Fig. 3 .

We would expect that, if the Ginzburg regime indeed produced enhanced decay of strings, then the string densities measured at later times should be smaller the longer the time the system spent within the range $T_{c} \geq T \geq T_{G}$.

Our results for the final string densities as a function of intermediate temperature $\epsilon_{i}$ and $\Delta t$ are summarized

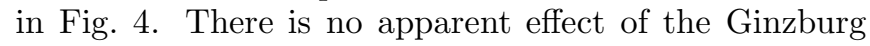
regime in reducing string densities at formation.

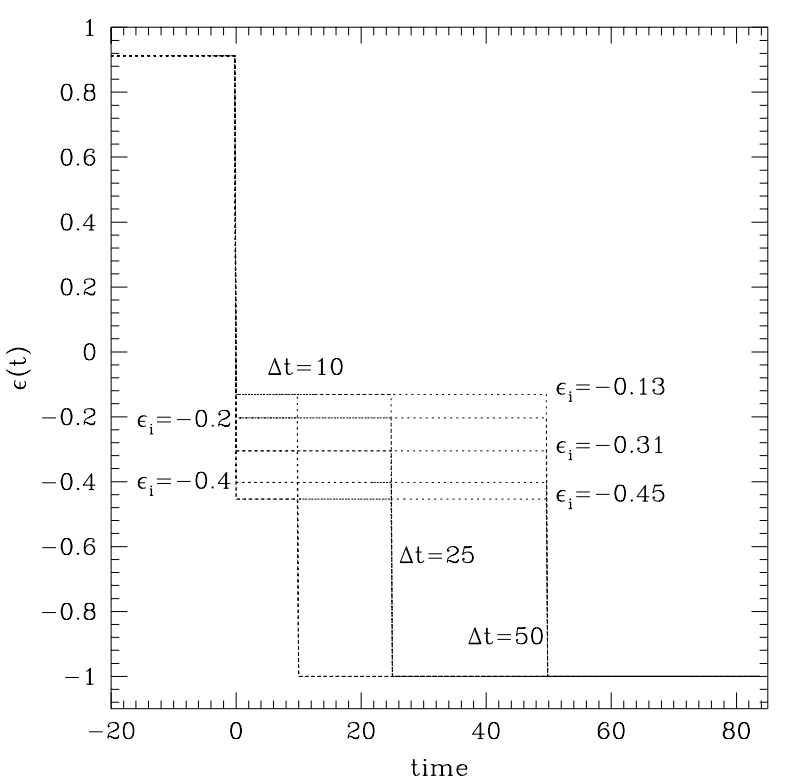

FIG. 3. Temperature trajectories for testing the effect of exposure to the Ginzburg regime on string densities. The system is first thermalized at a high temperature and then placed in contact with a heat bath at an intermediate temperature $\epsilon_{i}$ below $T_{c}$, for a time interval $\Delta t$.

If any trend is visible from Fig. A it is the opposite, namely a monotonic dependence of the final string densities on $\epsilon_{i}$ - the lower $\epsilon_{i}$, the less string is measured at later times.

The knowledge of the vortex string thermodynamics and of the time response of the fields in the critical domain again helps us understand this result. Strings and in particular long strings are inherited from high temperature (higher than $T_{c}$ ) topological fluctuations 10.

We can now use our knowledge of the Fokker-Planck solution to understand the observations of Fig. 4 . As we discussed above the small scales in the system equilibrate faster on a characteristic timescale $t \sim \eta^{-1}$. Small scale fluctuations dominate the thermal average in (9), which then allows us to take the effective value of $m^{2} \simeq m^{2}\left(T_{i}\right)$.

On the other hand, upon cooling through the critical point the large scales in the system display critical slowing down. This includes in particular the long strings in the sample which will be responsible for the signal at the time of measurement later. This slowing down leads to an imbalance in the string population out of equilibrium relative to their equilibrium counterpart, given by the existence of many more long strings.

This constitutes an excited state (described by $\mathcal{P}_{n \neq 0}$ ) relative to the true equilibrium of the system at intermediate temperatures below $T_{c}$. These states will then decay on a timescale $t_{e q}=E_{n}^{-1} \sim \eta / m^{2}\left(T_{i}\right)$. The value of $m^{2}\left(T_{i}\right)$ is smaller the closer $T_{i}$ is to $T_{c}$ and thus leads to a longer time scale for the decay of long strings. 


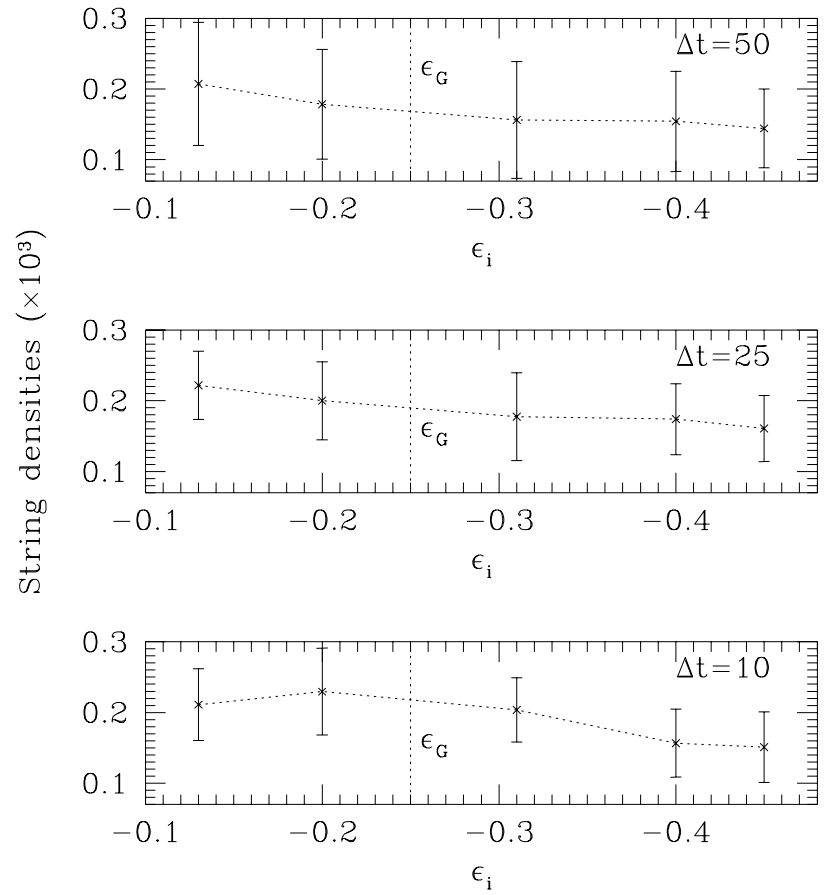

FIG. 4. The string density measured at the same later time $t>>t$ vs. intermediate temperature $\epsilon_{i}$. From top to bottom the three plots correspond to $\Delta t=10,20,50$, during which the system remained in contact with a heat bath at $T_{i}$. There is no visible role played by intermediate temperatures within the Ginzburg regime.

We can then predict a monotonic behavior for the string densities as observed in Fig. 4. At $T_{G}$ in particular $m^{2}=\lambda T_{G}^{2}$, by definition and $t_{e q} \sim \lambda T_{G}^{2} / \eta$.

Thus the conclusion is inescapable: The longer the time the system spends further from $T_{c}$ the less string it will display at later times where formation rates are measured.

\section{B. Memory of the Order Parameter Configuration near $T_{c}$}

An independent test on the possible role of thermal fluctuations in affecting string densities consists in reheating a quenched system to a temperature around $T_{c}$ (both below and above) and cooling it again at the same rate. This process tests the memory of the order parameter as well as that of other related quantities (see also [25]), such as defects.

The importance of this test is directly related to the canonical theory of defect formation as due to the critical dynamics of the fields. The final density of strings formed at the transition are then infered indirectly through the correlation length associated with the two-point correlator of the fields.

An example of the temperature $(\epsilon(t))$ trajectories used in testing the memory of the order parameter are shown in Fig. 同a.
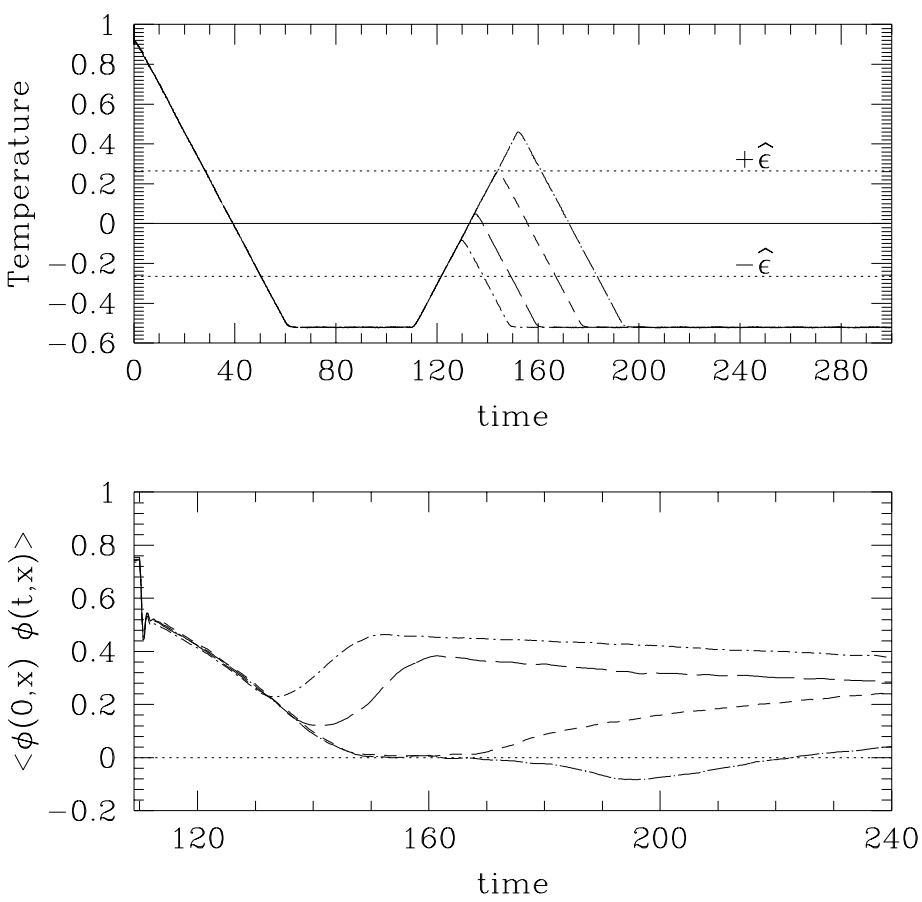

FIG. 5. a. Dependence of the bath temperature $\epsilon$ in time. After being quenched in temperature $\left(\tau_{Q}=80\right)$ the system is reheated at the same rate to a temperature $\epsilon_{f}=0.469,0.256,0.061,-0.068$ (top to bottom) and cooled again. b. The correlation function between the field at the time just before reheating and at later times, $\left\langle\phi_{i}\left(t_{\mathrm{rh}}, x\right) \phi_{i}\left(t+t_{\mathrm{rh}}, x\right)\right\rangle$ is plotted. There is a universal short time transient for the decorrelation of the field over small scales while the long time tails of the correlation function describe change over the mean fields. All four trajectories cross the Ginzburg regime, but only those reaching or crossing $+\hat{\epsilon}$ display a significant memory loss.

We are particularly interested in investigating under what circumstances thermal fluctuations can affect the large scale configuration of the order parameter. In order to produce a quantitative test we define the unequal-time two-point correlation function

$$
\begin{aligned}
& \left\langle\phi\left(x, t_{\mathrm{rh}}\right) \phi\left(x, t+t_{\mathrm{rh}}\right)\right\rangle \\
& =N \sum_{j=1}^{2} \sum_{i} \phi_{j}\left(x_{i}, t_{\mathrm{rh}}\right) \phi_{j}\left(x_{i}, t+t_{\mathrm{rh}}\right),
\end{aligned}
$$

where $N$ is an irrelevant normalization factor. This correlator has several interesting properties. For short times it displays a characteristic time, which describes the decay of correlations over very small spatial scales. This is the initial transient in Fig. Fb. We verified that this time and the form of the correlation function is in agreement with the forms predicted from a Boltzmann distribution for the fields. For later times the residual correlation comes from the motion of the order parameter (the field volume 
average). This average can be either positive or negative but, if thermal, will converge to zero at and above $T_{c}$.

Now, we are interested in determining whether the final field configuration over large spatial scales is correlated to the configuration prior to reheating. Fig. 5 shows that only if one crosses $T_{c}$, by more than $+\hat{\epsilon}$, is the memory of the initial quenched configuration erased (see in particular the two trajectories reaching higher temperatures in comparison to the others). For these trajectories the field correlations reach zero and after reheating evolve to a value manifestly different from that prior to reheating.

For trajectories within the Ginzburg regime, that do not cross $T_{c}$, the change in the configuration of the order parameter as measured by Eq. (16) is small. In particular the field configuration existing before reheating is approximately recovered as the fields are cooled. The same is true for the string densities, including those of long strings.

Again we can understand these results using the tool developed in section II. The thermalization time, which is nothing else than the response time $\tau$ in the KibbleZurek scenario, for long-wave length modes in the system is given by

$$
t_{e q} \simeq \frac{\eta}{m^{2}(T)}
$$

We argued that it is a reasonable approximation to take $T$ to be the final temperature since the small scales in the system equilibrate much faster (provided $m(T)<\eta$ ).

Now $\hat{t}$, or equivalently $\hat{\epsilon}$, is defined as the time when the long wave-length modes in the system can first respond to a change in bath temperature linear in time. It is computed by equating the scaling of the response time $\tau(T)$

$$
\tau(\epsilon(T))=\frac{\eta}{m^{2} \epsilon^{\nu z}(T)}
$$

to a linear change in time, imposed externally, i.e.

$$
\tau(\epsilon(\hat{t}))=\frac{\eta}{m^{2} \epsilon^{\nu z}(\hat{t})}=\hat{t}
$$

$z$ is another critical exponent whose mean-field value is 2 , see [10]. This relation is usually solved by assuming a linear dependence in time for $\epsilon(t)=t / \tau_{Q}$, where $\tau_{q}$ is the rate of the external quench.

Explicit calculation of $\Delta m^{2}(T)$ in (9) for our classical Boltzmann ensemble shows that

$$
\Delta m^{2}(T) \simeq \frac{\lambda}{\pi^{2}} \Lambda T
$$

where $\Lambda$ is the ultraviolet cut off. This cutoff has physical meaning and is related to the breakdown of our scalar field model for high energy excitations, eg. fermionic quasi-particles in ${ }^{3} \mathrm{He}$. If indeed the external bath temperature is changed linearly then $T$ in Eq. (20) can be taken, over time scales larger than $\eta^{-1}$, to be linear in time. It then results trivially that $\epsilon(t)$ is also linear, which validates our assumption.

The significance of $\hat{t}$ is that only when $|\epsilon(t)|>\hat{\epsilon}$ can the long wave-length modes in our system thermalize under an externally changing temperature at a rate $\tau_{Q}$, i.e. keep pace with the externally imposed changes. Due to theoretical uncertainties the value of $\hat{\epsilon}$ adopted in Fig. 5 a was measured by monitoring the response of the system directly. Details are described elsewhere [10.

At the initial time, for temperature trajectories of Fig. 5, the system is in the process of breaking the $U(1)$ symmetry spontaneously, i.e. the expectation value of the $k=0$ mode of $\phi,\langle\phi\rangle$ is non-zero. Then, as the system is heated towards $T_{c}$ equilibration of the long wave-length modes means that $\langle\phi\rangle \rightarrow 0$ and upon cooling show zero correlation in Fig. 5b to its initial state. Since thermalization of $\langle\phi\rangle$ can only occur for $\epsilon \geq \hat{\epsilon}$, only the temperature trajectories crossing $+\hat{\epsilon}$ can attain zero correlations.

It is then clear that the Ginzburg regime cannot change the symmetry breaking process of the system, including its associated long string configurations, unless a long amount of time is allowed. The Ginzburg regime is therefore less efficient at destroying topological defects (in the sense of requiring a longer time) than any other temperature range outside the critical domain.

\section{DISCUSSION AND CONCLUSIONS}

In this paper we have performed the most extensive analysis to date of the effects of large thermal fluctuations, within the Ginzburg regime, on the formation of topological defects. Our model field theory has already been studied extensively both in equilibrium and in tests of the theory of defect formation at temperature quenches, as predicted by the critical dynamics of the theory.

Under these controlled circumstances we analyzed critically the assumptions underlying the traditional argument for the Ginzburg temperature as the energy scale at which topological defects are formed. We then proceeded to show that the effects of thermal fluctuations in the Ginzburg regime upon a population of topological defects formed by the critical dynamics of the theory carries no particular signature and leads mostly to small qualitative changes in the defect densities predicted by the theory of defect formation.

We have also shown that even prolonged exposure of a quenched field configuration to the Ginzburg regime has little consequences in changing the order parameter configurations emerging at $-\hat{\epsilon}$, and associated string densities. In addition we established that to truly destroy a quenched field configuration existing below $-\hat{\epsilon}$, one has to expose the system to temperatures well above $T_{c}$. In particular for a linear quench trajectory, a temperature of $\epsilon \sim+\hat{\epsilon}$, must be reached in order to erase memory of the initial configuration. 
These results were confirmed by analytical arguments based on the solutions of the associated Fokker-Plank equation. This analysis supports the conclusion that given the same amount of time of exposure to a thermal bath at a given temperature, the Ginzburg regime is actually the least efficient range of temperatures at destroying the pattern of symmetry breaking inherited from criticallity. This includes topological defect configurations.

Our results fully support the theory of defect formation resulting from the critical dynamics of second order transitions [5] and all known thermodynamic results for vortex strings in $O(N)$ theories $13,15,12]$. In face of this evidence we are lead to conclude that arguments singling out a special energy scale $T_{G} \neq T_{c}$, which would play an important role in defect formation rely on assumptions that are not realized in the true (thermo)dynamics of our model and are thus invalid.

Thus we expect the results of this paper to carry over to the new Lancaster ${ }^{4} \mathrm{He}$ experiments. The results of reported in [9] in these experiments cannot therefore be attributed to the effects of Ginzburg regime in ${ }^{4} \mathrm{He}$.

\section{ACKNOWLEDGMENTS}

We thank T. Kibble, P. Laguna and R. Rivers for useful discussions. Numerical work was done on the Tdivision/CNLS Avalon Beowulf cluster, LANL. This research was supported by the U.S. Department of Energy, under contract W-7405-ENG-36.

[1] V.L. Ginzburg, Sov. Phys. Solid State 2, 1824 (1960).

[2] T.W.B. Kibble, J. Phys. A 9, 1387 (1976).

[3] T.W.B. Kibble, Phys. Rep. 67, 183 (1980).

[4] A. Vilenkin and E.P.S. Shellard, Cosmic Strings and other topological defects, (Cambridge University Press, Cambridge, U.K.,1994).

[5] W.H. Zurek, Acta Physica Polonica B 24 1301(1993); Nature 317505 (1995); Phys. Rep. 276177 (1996).

[6] C. Baüerle et al., Nature 382, 332 (1996).

[7] V.M.H. Ruutu et al., Nature 382, 334 (1996) ; V.M.H. Ruutu et al., Phys. Rev. Lett. 80, 1465 (1998).

[8] P.C. Hendry et al., Nature 368, 315 (1994).

[9] M. E. Dodd et al., Phys. Rev. Lett., 81, 3703 (1998).

[10] N.D. Antunes, L.M.A. Bettencourt, and W.H. Zurek, Phys. Rev. Lett. 822824 (1999).

[11] G. Karra and R. J. Rivers (1998), Phys. Rev. Lett. 81, 3707 (1998).

[12] G. Kohring, R. E. Shrock and P. Wills, Phys. Rev. Lett. 57, 1358 (1986).

[13] N.D. Antunes, L.M.A. Bettencourt and M. Hindmarsh, Phys. Rev. Lett. 80908 (1998).
[14] N.D. Antunes, and L.M.A. Bettencourt, Phys. Rev. Lett. 813083 (1998).

[15] A.K. Nguyen, A. Sudbo, Phys. Rev. B 582802 (1998).

[16] See eg. J. Zinn-Justin, Quantum field theory and critical phenomena, (Clarendon Press, Oxford, U.K., 1996).

[17] L.M.A. Bettencourt, unpublished.

[18] J. Kosterlitz and D. Thouless, J.Phys. C 6, 1181 (1973); J. Kosterlitz J.Phys. C 7, 1046 (1974).

[19] See G. A. Williams, Phys. Rev. Lett. 821201 (1999) and references therein.

[20] K. Kajantie et al., Phys.Lett. B 428334 (1998).

[21] E. J. Copeland et al., Physica A 179, 507 (1991).

[22] D. Mitchell and N. Turok, Phys. Rev. Lett. 581577 (1987).

[23] See eg. Electroweak Physics and the Early Universe, Eds. J.C. Romão and F. Freire, (Plenum Press, New York, 1994).

[24] L.M.A. Bettencourt, Phys. Lett. B 356297 (1995).

[25] A. Yates A. and W.H. Zurek, Phys. Rev. Lett. 80, 5477 (1998). 